\title{
Driver Drowsiness Detection and Alert System
}

\author{
Swapnil Titare ${ }^{1}$, Shubham Chinchghare ${ }^{1}$, K. N. Hande ${ }^{2}$
}

${ }^{1}$ Department of Computer Science and Engineering, Priyadarshini Bhagwati College of Engineering, Nagpur, Maharashtra, India

${ }^{2}$ Head of the Department, Department of Computer Science and Engineering, Priyadarshini Bhagwati College of Engineering, Nagpur, Maharashtra, India

\section{Article Info}

Volume 7, Issue 3

Page Number: 583-588

Publication Issue :

May-June-2021

\section{Article History}

Accepted : 18 June 2021

Published : 26 June 2021

\section{ABSTRACT}

Nowadays, accidents occur during drowsy road trips and increase day by day; It is a known fact that many accidents occur due to driver fatigue and sometimes inattention, this research is primarily devoted to maximizing efforts to identify drowsiness. State of the driver under real driving conditions. The aim of driver drowsiness detection systems is to try to reduce these traffic accidents. The secondary data collected focuses on previous research on systems for detecting drowsiness and several methods have been used to detect drowsiness or inattentive driving.Our goal is to provide an interface where the program can automatically detect the driver's drowsiness and detect it in the event of an accident by using the image of a person captured by the webcam and examining how this information can be used to improve driving safety can be used. . a vehicle safety project that helps prevent accidents caused by the driver's sleep. Basically, you're collecting a human image from the webcam and exploring how that information could be used to improve driving safety. Collect images from the live webcam stream and apply machine learning algorithm to the image and recognize the drowsy driver or not. When the driver is sleepy, it plays the buzzer alarm and increases the buzzer sound. If the driver doesn't wake up, they'll send a text message and email to their family members about their situation. Hence, this utility goes beyond the problem of detecting drowsiness while driving. Eye extraction, face extraction with dlib.

Keywords: Eye extraction, Dlib, Facial Extraction, Drowsiness, Machine Learning, EAR, Python, Face Detection

\section{INTRODUCTION}

The car accident is the leading cause of death, killing around 1.3 million people each year. Most of these accidents are caused by driver distraction or drowsiness. Drowsiness decreases the driver's concentration, activity, alertness, and alertness, and causes the driver to make slow decisions and sometimes not make decisions. Drowsiness affects mental alertness and reduces the driver's ability to drive a vehicle safely and increases the risk of human error, which can lead to death and injury [5]. the 
error rate for the driver had decreased. Countless people drive long distances on the road day and night. Lack of sleep or distractions such as talking on the phone, talking to the passenger, etc. can cause an accident. To avoid these accidents, we propose a system that will warn the driver if they are distracted or drowsy.

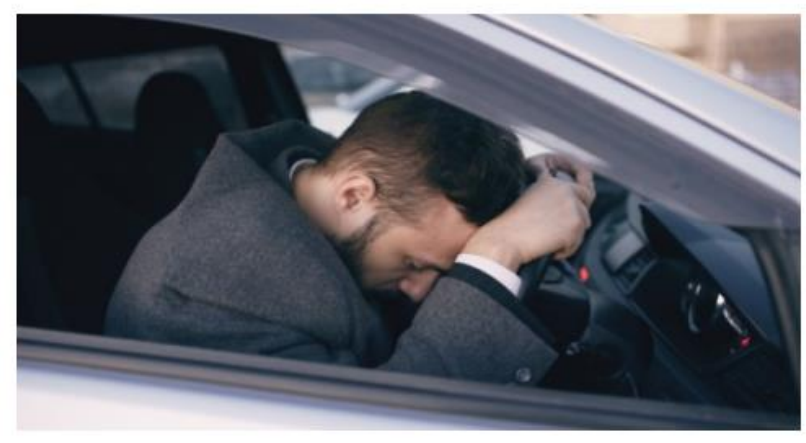

Fig: Drowsy Driver

Face and brand recognition is used with the help of image processing of facial images captured by the camera to identify distractions or drowsiness. To solve the problem, we came up with the implemented solution in the form of image processing. Perform image editing. , OpenCV and Dlib open source libraries are used. Python is employed because the language to implement the idea. associate degree infrared camera is used to endlessly track the driving force' facial markings and eye movements. This project mainly focuses on the driver's eye markings. Driver. Eye characteristics are continuously tracked to detect drowsiness. Images are captured by the camera, these images are forwarded to an image processing module that performs face recognition to detect distraction and drowsiness of the driver. sThe following use cases are covered in this project. If the driver's eyes are closed for a limited period of time, the driver is considered drowsy and the corresponding audible alarm is used to warn the driver.

\section{METHODS AND MATERIAL}

\section{Tools \& Image Processing Methods}

Open CV: OpenCV (Open-Source Computer Vision) is the Swiss Army Knife of Computer Vision, it has a wide range of modules that can help us with many Computer Vision problems, but perhaps the most useful part of OpenCV is its architecture. and memory management. It gives you a framework in which to work with pictures and videos however you want, using OpenCV algorithms or your own, without worrying about allocating and reallocating memory for your pictures. optimized and can be used for real-time video and image processing The highly optimized image processing function of OPENCV is used by the author for real-time image processing of live video streaming from the camera.

DLib: Dlib is a modern C toolkit with algorithms and tools for machine learning to create complex $\mathrm{C}++$ software to solve real problems. It is used in a wide variety of fields in both industry and academia, including robotics, embedded devices, cell phones, and large, high-performance computing environments. Lib's open source licenses allow you to use it in any application for free.The author uses the open source Dib library for the CNN (Neural Networks) implementation. The author uses highly optimized prediction functions and detectors of previously learned face shapes to detect facial features.

\section{EAR (Eye Aspect Ratio)}

The numerator of this equation calculates the distance between the vertical landmarks of the eye, while the denominator denotes. calculates distance between the horizontal eye reference points, 
weighting the denominator accordingly since there is only one. The aspect ratio of the eye is roughly constant when the eye is open, but quickly drops to zero when you blink. When the person blinks, the aspect ratio of the eyes drops dramatically and approaches zero. As shown in Figure 2, the aspect ratio of the eyes is constant, then quickly drops to zero and then increases again, suggesting that a single blink has occurred.

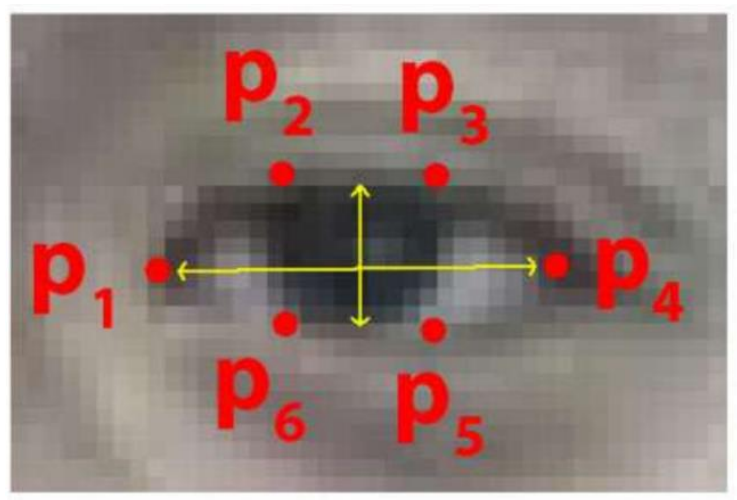

Fig : Eyes Points

\section{Face Recognition}

The following sections describe the face recognition algorithms Eigenface, Fisherface, Histogram of Local Binary Pattern and their implementation in OpenCV: Histogram of Local Binary Pattern (LBPH) Local binary patterns were used as classifiers in Computer Vision and 1990 by Li. suggested Wang [4] The combination of LBP with histogram-oriented gradients was introduced in 2009, which improved the performance in certain data sets [5]. For feature coding, the image is divided into cells (4 $\mathrm{x} 4$ pixels) using a surrounding pixel clockwise or counterclockwise. The values are compared with the central ones shown in Figure 6. The intensity or brightness value of each neighbor is compared to the central pixel. Depending on whether the difference is greater or less than 0 , the location is assigned a 1 or 0 . an 8-bit value for the cell.The advantage of this technique is that even if the brightness of the image is .

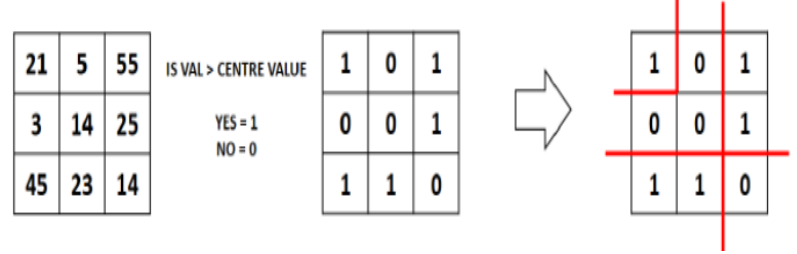

Fig: - LBPH

In Figure, the result will be the same as before. in larger cells to determine the frequency of occurrence of values, which speeds up the process. By analyzing the results in the cell, edges can be identified as the values change. By calculating the values for all cells and concatenating the histograms, feature vectors can be obtained. The input images are classified according to the same procedure and compared with the data set, and the distance is determined. By setting a threshold, you can tell if the face is familiar or unfamiliar.Eigenface and Fisherface calculate the dominant features of the entire training set, while LBPH analyzes them individually.

\section{Algorithm Steps}

Step 1 - Take image as input from a camera.

Step 2 - Recognize the face in the image and create a region of interest (ROI).

Step 3 -Recognize the eyes from the ROI and send them to the classifier

Step 4 - The classifier classifies whether the eyes are open or closed

Step 5 - Calculate the score to be verified. when the person is sleepy. 


\section{Flowchart}

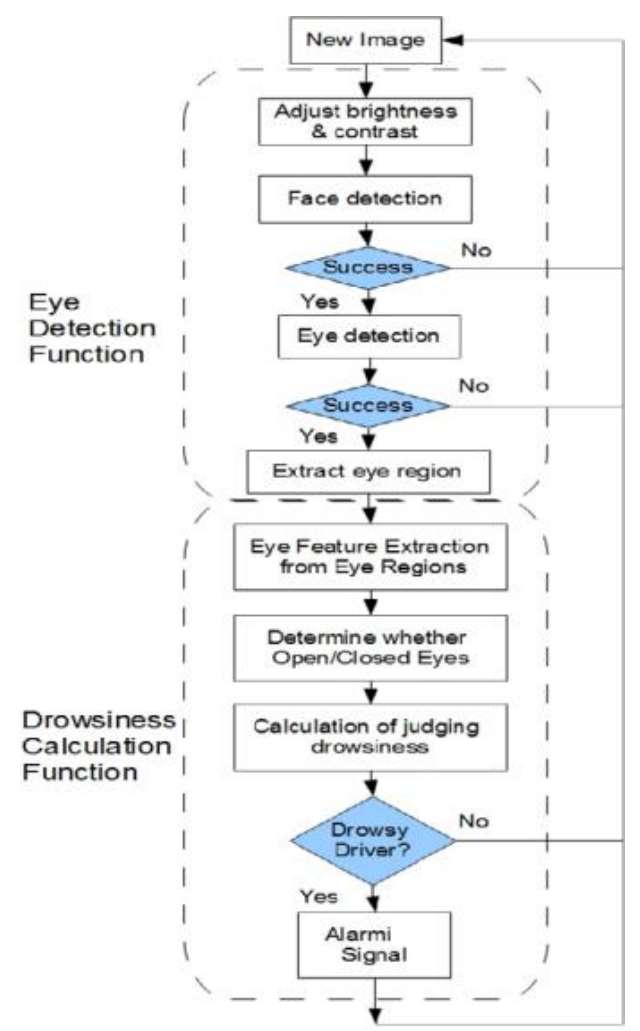

Fig: Drowsiness Detection

With a webcam we take pictures as input. To access the webcam, we created an infinite loop that captures each frame. We will use the method provided by OpenCV to access the camera and configure the capture object, we will read each frame and store the image in a frame variable. In order to recognize the face in the image, we must first convert the image to grayscale, as the OpenCV algorithm for object recognition uses gray images as it is input. We don't need any color information to recognize the objects. We use a hair cascade classifier to identify faces. Then we do face recognition. Returns an array of detections with $\mathrm{x}, \mathrm{y}$ coordinates and the height and width of the bounding box of the object. Now we can iterate over the faces. and draw contour boxes for each face.

\section{III.RESULTS AND DISCUSSION}

In this system we have divide into several modules shown below

1. Login Module - In this module user will able to enter in system and available to start the drowsiness system and system will start the camera and start monitoring the driver. In this module user has to provide there credentials such as username and password.

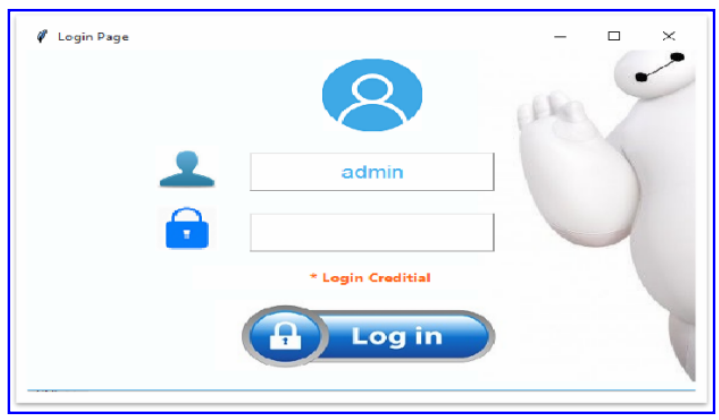

Fig : Login Page

2. Registration Module - In this module user able to register his details such as its contact number, email and also his family member details and their number and emails which going to use to make them alert by sending email and SMS in stage of drowsiness.

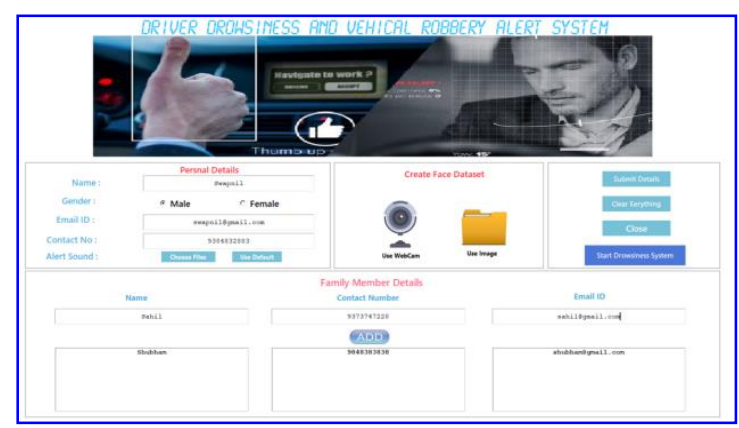

Fig : Registration Page

3. Eye Extraction Module -In this module it will detect the eyes and face landmarks from live webcam feed and apply algorithms on image to detect driver drowsy or not. 


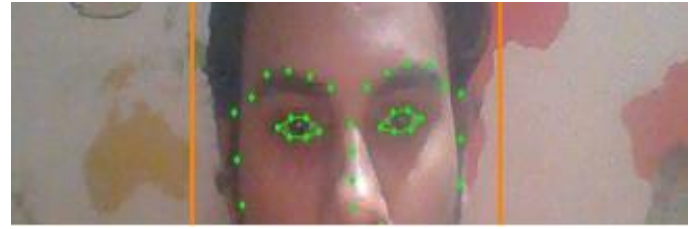

Fig: Eye Extraction

4. Drowsiness Detector Module - In this module it will detect the eyes from live webcam feed and apply algorithms on image to detect driver drowsy or not.

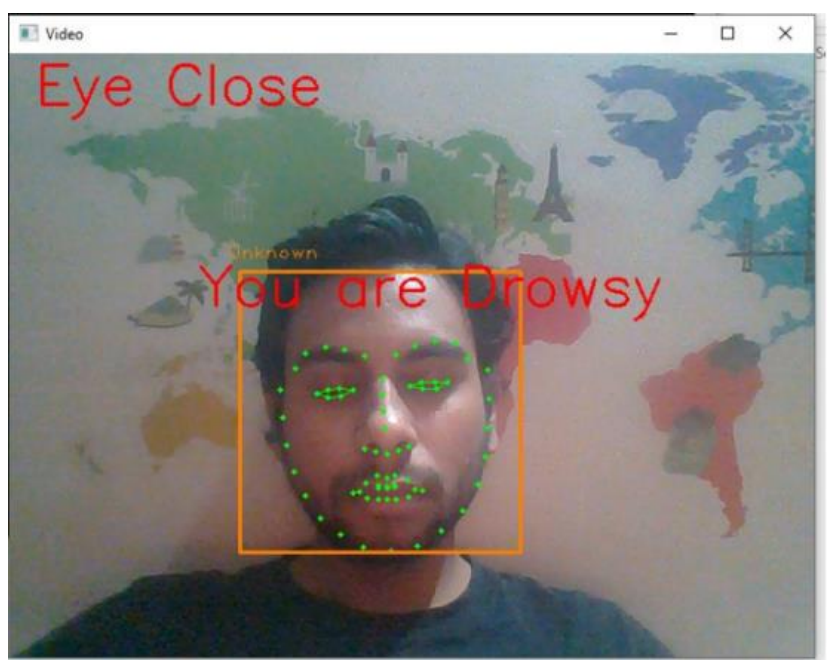

Fig Drowsiness Detection

5. Face Identification Module - In this module it will going to detect the driver identification with the help of face recognition method and with this authentication it will fetch the driver family details from database and sent alert message.

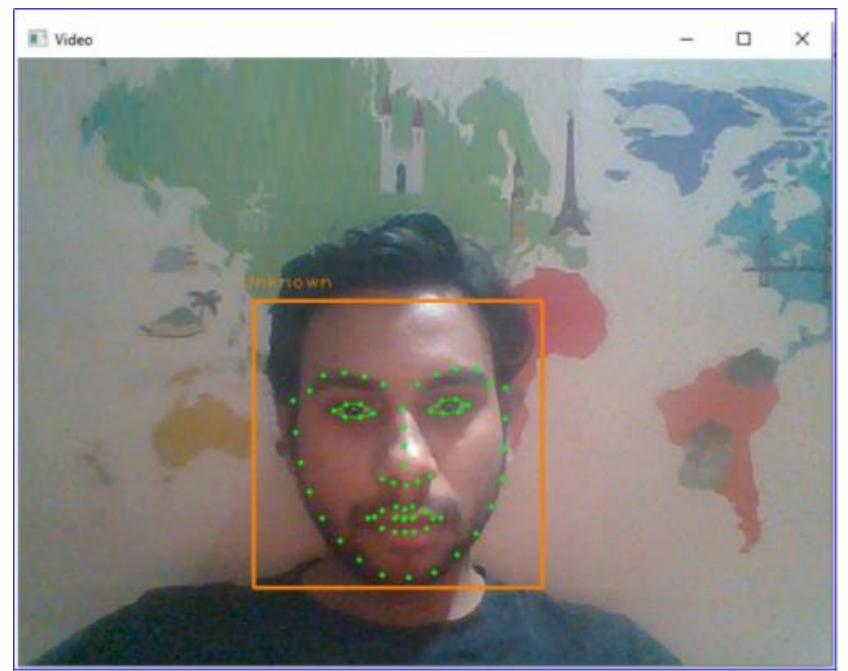

Fig : Face Identification

6. Alert Module - If driver will not wake up in $\mathbf{5 0}$ alerts alarm music then it send SMS and email to user family member to inform them that you are drowsy along with its current photo and location.

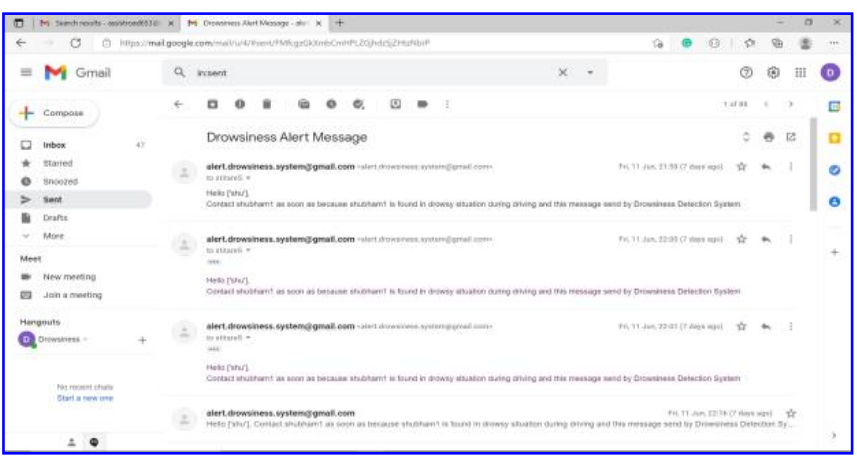

Fig : Alert Message

\section{Requirement}

\section{Software Requirement}

1. Front End : Tkinter (Page)

2. Back End : Python

3. Domain : Machine Learning,

4. Algorithm : LPBH, DLIB, HaarCascade.

\section{Hardware Requirement}

1. Processor : i3 or grater

2. RAM : 4GB or greater

3. Hard Disk : $50 \mathrm{~GB}$ or greater

4. Connectivity : LAN or WIFI, Camera

\section{IV.CONCLUSION}

The proposed gadget on this evaluation affords accurate detection of driving force fatigue. The evaluation and layout of driving force drowsiness detection gadget is presented. The proposed gadget is used to keep away from numerous avenue accidents due to drowsy using and it is able to additionally assist drivers to live wakeful whilst using via way of means of giving a caution whilst the driving force is 
sleepy.the precept concept of drowsiness detection device it detects and offer information of behavioural, vehicular and physiological parameters based totally on it. It seems that in the moments in advance than falling asleep, drivers yawn less, now no longer more, frequently. This highlights the significance of the use of examples of fatigue and drowsiness situations in which topics without a doubt fall sleep. despite the fact that the accuracy charge of using physiological measures to discover drowsiness is excessive, those are pretty intrusive. But this intrusive nature may be resolved via way of means of manner of the usage of contactless electrode placement. as a result, it would be really well worth fusing physiological measures, collectively with Dlib, with behavioural and carbased totally measures in the development of an green drowsiness detection device. further, it's far essential to bear in mind the the use of surroundings to obtain most useful effects.

\section{REFERENCES}

[1]. A Study of Heart Rate and Brain System Complexity and Their Interaction in SleepDeprived Subjects. Kokonozi A.K., Michail E.M., Chouvarda I.C., Maglaveras N.M. Bologna, Italy. : Computers in Cardiology, 2008.

[2]. Effects of partial and total sleep deprivation on driving performance. Peters R.D., Wagner E., Alicandri E., Fox J.E., Thomas M.L., Thorne D.R., Sing H.C., Balwinski S.M. 1999.

[3]. Effect of driving duration and partial sleep deprivation on subsequent alertness and performance of car drivers. Otmani S, Pebayle T, Roge J, Muzet A. 2005.

[4]. TemplateMatching. [Online] Apr 21, 2014 [Cited: Sep 8, 2014.] Real time drowsy driver detection using haarcascade samples. Dr.Suryaprasad J, Sandesh D, Saraswathi V.

[5]. Integrated Approach for Nonintrusive Detection of Driver Drowsiness. Yu, Xun. Duluth : s.n., 2012.
[6]. Rajneesh, "Real Time Drivers Drowsiness Detection and alert System by Measuring EAR," International Journal of Computer Applications (0975 - 8887) Volume 181 - No. 25, November2018 .

[7]. Jay D. Fuletra., “A Survey on Driver's Drowsiness Detection Techniques" International Journal on Recent and Innovation Trends in Computing and Communication ISSN: 2321-8169 Volume: 1 Issue: 11,2013 .

\section{Cite this article as :}

Swapnil Titare, Shubham Chinchghare, K. N. Hande, "Driver Drowsiness Detection and Alert System", International Journal of Scientific Research in Computer Science, Engineering and Information Technology (IJSRCSEIT), ISSN : 2456-3307, Volume 7, Issue 3, pp.583-588, May-June-2021. Available at doi : https://doi.org/10.32628/CSEIT2173171 Journal URL : https://ijsrcseit.com/CSEIT2173171 